\section{Fat deposition in the nuchal region is associated with insulin resistance and low metabolic clearance rate of glucose in Nigerian adult males}

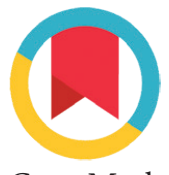

CrossMark

\author{
Kehinde Sola Akinlade, ${ }^{1 *}$ Abosede Olabimpe Ayinde, ${ }^{2}$ Sheu Kadiri Rahamon, ${ }^{2}$ \\ Rasaki Adegoke Sanusi ${ }^{3}$
}

\section{ABSTRACT}

Background: The role of classical proxies for obesity such as the body mass index (BMI) and waist circumference (WC) in the development of insulin resistance (IR) is well reported. However, there is the dearth of information on the association between subcutaneous fat deposition in the nuchal region and the development of IR.

Objective: To determine the risk of IR development posed by deposition of fat in the nuchal region of the body.

Methods: Fifty adult males with CFF and 50 males without CFF were enrolled into this study. Standard Oral Glucose Tolerance Testing (OGTT) was performed and fasting plasma glucose (FPG), 2 hours post prandial glucose (2hrPPG), lipids, fasting insulin ( $F \mathrm{I}), 2$ hours postprandial insulin (2hrPPI) levels were determined. Thereafter, indices of IR and

estimated metabolic clearance rate of glucose (eMCR) were calculated using standard formula.

Results: Levels of Fl, $2 \mathrm{hrPPI}$ and the median values of indices of IR were significantly higher while median values of indices of insulin sensitivity and $\mathrm{EMCR}$ were significantly lower in CFF compared with the controls. Predicting the diagnostic property of CFF for insulin sensitivity, CFF thickness has $69.5 \%$ comparability to the QUICKI cut-off values and a cut-off value of $12.5 \mathrm{~cm}$ of CFF thickness was found to have a sensitivity of $75.0 \%$ and a specificity of $55.3 \%$ in indicating Low Insulin Sensitivity (LIS). Conclusion: Adult males with CFF have IR and low eMCR and are thus, prone to developing cardiometabolic diseases. Also, CFF thickness appears to be a good anthropometric index of IR.
*Correspondence to:

Dr. K. S. Akinlade, Metabolic Research Unit, Department of Chemical Pathology, College of Medicine, University of Ibadan \& University College Hospital, Ibadan, Nigeria.

ksakinlade@yahoo.co.uk

Cite This Article: Akinlade, K.S., Ayinde, A.O., Rahamon, S.K., Sanusi, R.A. 2020. Fat deposition in the nuchal region is associated with insulin resistance and low metabolic clearance rate of glucose in Nigerian adult males. Diabesity 6(3): 19-24. DOl: $10.15562 /$ diabesity.2020.65

Key words: Cardiovascular diseases, Cervical fat fold, Insulin resistance, Metabolic clearance rate of glucose, Type 2 diabetes mellitus.

\section{INTRODUCTION}

Obesity plays a key role in the development of insulin resistance (IR). ${ }^{1}$ However, epidemiological and metabolic studies have shown that the common cardio-metabolic diseases including insulin resistance, dyslipidaemia, type 2 diabetes mellitus (T2DM), and cardiovascular diseases (CVDs) are more closely related to the distribution of body fat rather than the absolute degree of fatness. ${ }^{2,3}$ Several reports $^{3-5}$ have shown that visceral adipose tissue (VAT) is closely linked to an increasing incidence of insulin resistance, T2DM, and a higher risk of cardiovascular disease due to a high production of pro-inflammatory adipocytokines, oxidative stress, and renin-angiotensin-aldosterone system (RAAS) activation by VAT.

Similarly, indices of upper body fat accumulation such as neck circumference (NC) has been shown to be an independent predictor of cardiovascular disease risk beyond BMI and waist circumference (WC). Nielsens et al. ${ }^{6}$ reported that upper body fat is a major contributor to systemic free fatty acid (FFA) availability and hence, associated with IR, increased very-low-density lipoprotein cholesterol production, and endothelial cell dysfunction. The reports of Preis et al. ${ }^{7}$ and Stabe et al. ${ }^{8}$ showed that large neck circumference is associated with various cardio-metabolic disruptions even after adjusting for VAT and body mass index (BMI). These reports clearly show that accumulation of fat in the upper segment of the body such as the neck may confer risk above and beyond visceral abdominal fat accumulation.

Currently, a number of proxies of obesity are used as markers of CVD risk. These include the traditional body mass index (BMI), and indices of central adiposity such as waist circumference (WC), waist-to-hip ratio (WHR) and waist-toheight ratio (WHtR). ${ }^{1,-11}$ However, the use of indices of upper body fat accumulation as markers of IR and consequently, CVDs is presently not common as there is the dearth of information on various indices of fat accumulation derivable from the upper segment of the body that could be used as markers of cardio-metabolic diseases risk. This study was therefore designed to determine the risk

\footnotetext{
'Metabolic Research Unit, Department of Chemical Pathology, College of Medicine, University of Ibadan \& University College Hospital, Ibadan, Nigeria. ${ }^{2}$ Department of Chemical Pathology, College of Medicine, University of Ibadan, Nigeria.

${ }^{3}$ Department of Human Nutrition, College of Medicine, University of Ibadan, Nigeria.
} 
of IR development posed by deposition of fat in the nuchal region of the body.

\section{MATERIALS AND METHODS}

\section{Study participants}

A total of 100 apparently healthy participants comprising 50 adult males with Cervical Fat Fold (CFF) and 50 adult males without CFF were enrolled into this case-control pilot study. Individuals with any forms of neck deformities, already diagnosed with CVD or T2DM or on drugs that could alter glucose metabolism such as steroids and those on diuretics were excluded from this study.

\section{Ethical consideration}

Before the commencement of the study, the study was approved (UI/EC/17/0233) by the University of Ibadan/University College Hospital (UI/UCH) Joint Ethics Review Committee. Also, written informed consent was obtained from the study participants.

\section{Data collection}

A semi-structured questionnaire was used to obtain information on basic demographic and social characteristics, as well as medical history of the participants.

\section{Anthropometric and blood pressure measurements}

Height, body weight (BW), waist (WC) and hip (HC) circumference, and blood pressure were determined using standard measurement methods. Thereafter, BMI was calculated as body weight divided by the square of height $\left(\mathrm{kg} / \mathrm{m}^{2}\right)$, waist-tohip ratio (WHR) was calculated as waist circumference divided by the hip circumference. The neck circumference was measured with the head erect and eyes facing forward, using a flexible tape positioned horizontally at the upper margin of the laryngeal prominence. The thickness of the CFF was measured using skin fold thickness caliper to the nearest $0.1 \mathrm{~mm}$ at the midline while the participant was standing erect with head in horizontal position and shoulder relaxed.

\section{Blood sample collection}

Venous blood samples were collected from each participant after an overnight fast of about 10 12 hours and at 120 min after a standard 75-g Oral Glucose Tolerance Test (OGTT) was performed. Samples obtained were dispensed into fluoride oxalate, plain and EDTA bottles for glucose, insulin and lipid profile analysis respectively. Thereafter, serum/plasma was obtained appropriately and stored at $-20^{\circ} \mathrm{C}$ until analyzed.

\section{Laboratory analyses}

Glucose oxidase method was used to determine the plasma level of glucose while insulin level was determined using ELISA (Genway Biotech Inc., San Diego, USA). The plasma levels of HDL-C, TG and TC were determined using enzymatic methods while the Frieldwald et al. ${ }^{12}$ formula was used to calculate the LDL-C level.

\section{Calculation of indices of insulin resistance/ sensitivity}

a) Homeostasis model assessment-estimated insulin resistance (HOMA-IR) was calculated as the product of fasting insulin $(\mu \mathrm{U} / \mathrm{ml})$ and fasting glucose $(\mathrm{mmol} / \mathrm{l})$ divided by $22.5{ }^{13}$

b) Quantitative Insulin Sensitivity Check Index (QUICKI) was calculated as $1 /$ [log fasting insulin $(\mu \mathrm{U} / \mathrm{ml})+\log$ fasting glucose $(\mathrm{mg} / \mathrm{dl})] .{ }^{13}$

c) Fasting insulin resistance index (FIRI) was calculated as [fasting glucose $(\mathrm{mg} / \mathrm{dl})+$ fasting insulin $(\mu \mathrm{U} / \mathrm{ml})] / 25$.

d) Glucose-insulin ratio (G/I) was calculated by diving fasting glucose $(\mathrm{mg} / \mathrm{dl})$ by fasting insu$\operatorname{lin}(\mu \mathrm{U} / \mathrm{ml}) .^{13}$

e) Estimated insulin sensitivity index $\left(\mu \mathrm{mol} . \mathrm{kg}^{-1}\right.$. $\left.\mathrm{min}^{-1} \cdot \mathrm{pmol} / \mathrm{l}\right)$ was calculated as $0.222-0.00333 \times$ BMI $-0.0000779 \times \mathrm{I}_{120}-0.000422 \times$ Age. $^{14}$

f) Estimated metabolic clearance rate of glucose $\left(\mathrm{ml} \cdot \mathrm{kg}^{-1} \cdot \mathrm{min}^{-1}\right)$ was calculated as 19.240 0.281 x BMI $-0.00498 \times \mathrm{I}_{120}-0.333 \times \mathrm{G}_{120}{ }^{14}$

\section{Statistical Analysis}

Statistical analysis was carried out using SPSS statistical software version 23.0 for windows. The Gaussian distribution of the variables was assessed using histogram with normal curve. Differences in mean of variables with Gaussian distribution was determined using the independent Student's t-test while differences in medians of variables with non-Gaussian distribution was determined using the Mann-Whitney $U$ test. In order to find out if OFF thickness could predict insulin sensitivity status, the Area Under the Receiver Operating Characteristic Curve (AUROC) was determined using QUICKI cut-off values of $\geq 0.31$ and $\leq 0.30$ which depict Normal Insulin Sensitivity (NIS) and Low Insulin Sensitivity (LIS). ${ }^{15}$ Spearman correlation was used to determine the correlation between variables. All tests were 2 -tailed and $P<0.05$ was considered as statistically significant.

\section{RESULTS}

The mean (standard deviation) and median (interquartile range) thickness of CFF in the cases were $12.81 \mathrm{~cm}(5.55 \mathrm{~cm})$ and $13.00 \mathrm{~cm}(8.00 \mathrm{~cm}-$ $16.25 \mathrm{~cm}$ ) respectively. 
As shown in Table 1, the mean BW, BMI, WC, HC, WHR and neck circumference were significantly higher in CFF compared with the controls. However, no significant differences were observed in the mean levels of FPG, 2hrPPG and lipid profile when CFF was compared with the controls. Although the differences were not significantly different, the mean systolic BP and diastolic BP were higher in OFF compared with the controls.

Differences in insulin resistance/sensitivity status of the study participants are shown in Figures 1 and 2. Males with CFF have IR and low insulin sensitivity (LIS) status. The median levels of $2 \mathrm{hrPPI}, \mathrm{FI}$ and indices of insulin resistance (HOMA-IR and FIRI) were significantly higher while indices of insulin sensitivity (QUICKI, 1/ FI, eISI) were significantly lower in CFF compared with the controls.
Poor glucose disposal rate was also observed in males with CFF as eMCR was significantly lower in CFF compared with the controls (Figure 3).

The correlation between CFF thickness and selected metabolic parameters are shown in Table 2. CFF thickness had significant positive correlation with important anthropometric indices including body weight, BMI, WC and HC. Similarly, insulin resistance increases as $\mathrm{CFF}$ thickness increases as demonstrated by the significant positive correlation between CFF thickness and indices of insulin resistance (HOMA-IR and FIRI) (Table 2). In contrast, insulin sensitivity decreases as CFF thickness increases as demonstrated by the significant negative correlation between CFF thickness and QUICKI; an index of insulin sensitivity (Table 2).

As shown in Table 3 and Figure 4, it was observed that CFF thickness has $69.5 \%$ comparability to the QUICKI cut-off values. A cut-off value of $12.5 \mathrm{~cm}$

Table 1 Age, anthropometric indices, metabolic factors and indices of insulin resistance in participants with CFF and controls

\begin{tabular}{|c|c|c|c|}
\hline Parameters & CFF $(n=50)$ & Controls $(n=50)$ & $P$-value \\
\hline Age (years) & $48.16 \pm 13.13$ & $49.92 \pm 14.40$ & 0.525 \\
\hline Height (m) & $1.71 \pm 0.08$ & $1.68 \pm 0.07$ & $0.037^{\star}$ \\
\hline Body weight (kg) & $81.44 \pm 13.83$ & $60.46 \pm 9.22$ & $0.000^{*}$ \\
\hline BMI $\left(\mathrm{kg} / \mathrm{m}^{2}\right)$ & $27.97 \pm 4.59$ & $21.46 \pm 2.77$ & $0.000^{*}$ \\
\hline $\mathrm{WC}(\mathrm{cm})$ & $95.98 \pm 12.40$ & $79.05 \pm 8.47$ & $0.000^{*}$ \\
\hline $\mathrm{HC}(\mathrm{cm})$ & $102.60 \pm 9.73$ & $90.38 \pm 7.02$ & $0.000^{\star}$ \\
\hline WHR & $0.93 \pm 0.05$ & $0.87 \pm 0.06$ & $0.000^{*}$ \\
\hline $\mathrm{NC}(\mathrm{cm})$ & $38.96 \pm 2.60$ & $35.11 \pm 1.88$ & $0.000^{*}$ \\
\hline $\mathrm{SBP}(\mathrm{mmHg})$ & $130.02 \pm 21.85$ & $125.20 \pm 20.34$ & 0.256 \\
\hline DBP (mmHg) & $81.44 \pm 14.82$ & $78.82 \pm 11.52$ & 0.326 \\
\hline Pulse (mmHg) & $71.74 \pm 9.18$ & $70.68 \pm 10.90$ & 0.600 \\
\hline FPG (mg/dl) & $92.24 \pm 15.69$ & $89.24 \pm 10.43$ & 0.263 \\
\hline 2hrPPG (mg/dl) & $104.40 \pm 26.58$ & $99.22 \pm 14.25$ & 0.227 \\
\hline $\mathrm{TC}(\mathrm{mg} / \mathrm{dl})$ & $177.96 \pm 26.55$ & $178.00 \pm 30.78$ & 0.994 \\
\hline TG (mg/dl) & $137.10 \pm 34.61$ & $130.30 \pm 37.61$ & 0.349 \\
\hline $\mathrm{HDL}(\mathrm{mg} / \mathrm{dl})$ & $57.86 \pm 11.41$ & $53.50 \pm 13.20$ & 0.080 \\
\hline LDL (mg/dl) & $92.49 \pm 21.08$ & $97.28 \pm 22.02$ & 0.272 \\
\hline $\mathrm{FI}(\mu \mathrm{U} / \mathrm{ml})$ & $9.66(4.38-17.88)$ & $3.42(2.34-6.22)$ & $0.000^{*}$ \\
\hline $2 \mathrm{hrPPI}(\mu \mathrm{U} / \mathrm{ml})$ & $58.17(16.83-84.69)$ & $18.75(7.79-41.09)$ & $0.000^{\star}$ \\
\hline $1 / \mathrm{FI}$ & $0.10(0.06-0.23)$ & $0.29(0.16-0.43)$ & $0.000^{\star}$ \\
\hline FIRI & $35.49(18.26-64.09)$ & $12.00(7.92-21.87)$ & $0.000^{\star}$ \\
\hline TG-HDL ratio & $2.35(1.89-2.96)$ & $2.58(1.82-3.34)$ & 0.652 \\
\hline eISI & $0.07(0.05-0.10)$ & $0.12(0.11-0.13)$ & $0.000^{*}$ \\
\hline
\end{tabular}

Results are presented as mean \pm standard deviation or median (interquartile range), ${ }^{*}$ Significant at $\mathrm{p}<0.05, \mathrm{BMI}=$ Body mass index, $\mathrm{WC}=$ Waist circumference, $\mathrm{HC}=$ Hip circumference, $\mathrm{WHR}=$ Waist-hip ratio, $\mathrm{NC}=$ Neck circumference, $\mathrm{CFF}=\mathrm{Cervical}$ fat fold thickness, $\mathrm{SBP}=$ Systolic blood pressure, $\mathrm{DBP}=$ Diastolic blood pressure, $\mathrm{FPG}=$ Fasting plasma glucose, $2 \mathrm{hrPPG}=2 \mathrm{hour}$ postprandial glucose, $\mathrm{TC}=$ Total cholesterol, $\mathrm{TG}=$ Triglyceride, $\mathrm{HDL}=$ High density lipoprotein-cholesterol, $\mathrm{LDL}=$ Low density lipoprotein-cholesterol, FI = Fasting serum insulin, $2 \mathrm{hrPPI}=2$ hour postprandial insulin, FIRI $=$ Fasting insulin resistance index, eISI = Estimated insulin sensitivity index 
Table 2 Correlation between CFF thickness with metabolic parameters in participants with CFF

\begin{tabular}{lcc}
\hline CFF $(\mathbf{c m})$ & r-value & $P$-value \\
\hline Body weight $(\mathrm{kg})$ & 0.412 & $0.003^{*}$ \\
BMI $\left(\mathrm{kg} / \mathrm{m}^{2}\right)$ & 0.509 & $0.000^{*}$ \\
WC $(\mathrm{cm})$ & 0.513 & $0.000^{*}$ \\
HC $(\mathrm{cm})$ & 0.391 & $0.005^{*}$ \\
NC $(\mathrm{cm})$ & 0.505 & $0.000^{*}$ \\
HOMA-IR & 0.367 & $0.009^{*}$ \\
FIRI & 0.367 & $0.009^{*}$ \\
QUICKI & -0.367 & $0.009^{*}$ \\
\hline
\end{tabular}

${ }^{*}$ Significant at $\mathrm{p}<0.05, \mathrm{CFF}=$ Cervical fat fold thickness, $\mathrm{BMI}=$ Body mass index, $\mathrm{WC}=$ Waist circumference, $\mathrm{HC}=$ Hip circumference, $\mathrm{NC}=$ Neck circumference, HOMA-IR $=$ Homeostasis model assessment-estimated insulin resistance, FIRI $=$ Fasting insulin resistance index, QUICKI $=$ Quantitative Insulin Sensitivity Check Index

Table 3 Area Under the Curve of CFF thickness in men with Normal Insulin Sensitivity status and men with Low Insulin Sensitivity status

\begin{tabular}{lcccc}
\hline & & \multicolumn{2}{c}{$95 \%$ Confidence Interval } \\
\cline { 4 - 5 } AUROC & Standard Error & $P$-value & Lower Boundary & Upper Boundary \\
\hline 0.695 & 0.085 & $0.043^{*}$ & 0.528 & 0.862 \\
\hline
\end{tabular}

${ }^{\star}$ Significant at $\mathrm{p}<0.05$

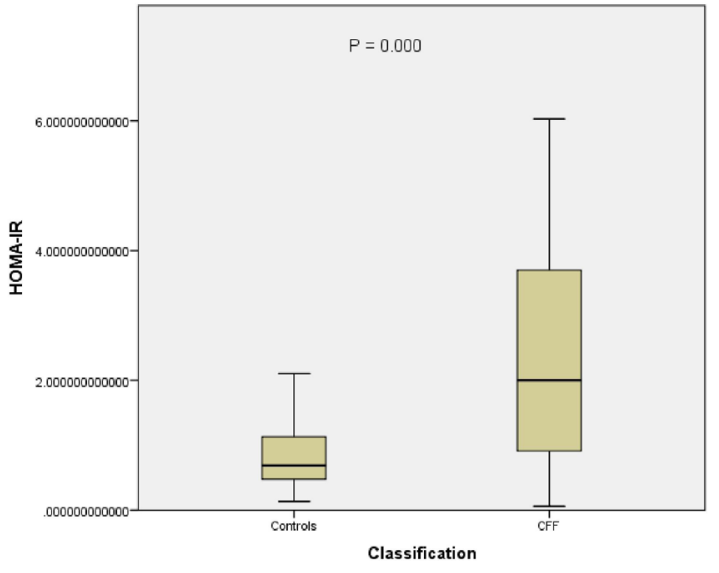

Figure 1 Homeostasis model assessment-estimated insulin resistance (HOMA-IR) in CFF and controls

of CFF thickness was found to have a sensitivity of $75.0 \%$ and a specificity of $55.3 \%$ in indicating LIS.

\section{DISCUSSION}

Evidences are abound indicating that IR is an independent risk factor for T2DM, CVDs, hypertension, dyslipidaemia and certain types of cancer. ${ }^{16-18}$ The global continuous rise in the prevalence of IR associated diseases therefore, indicates that there is the need for identification of risk factors for IR that can easily be utilized especially, in poor resource countries.

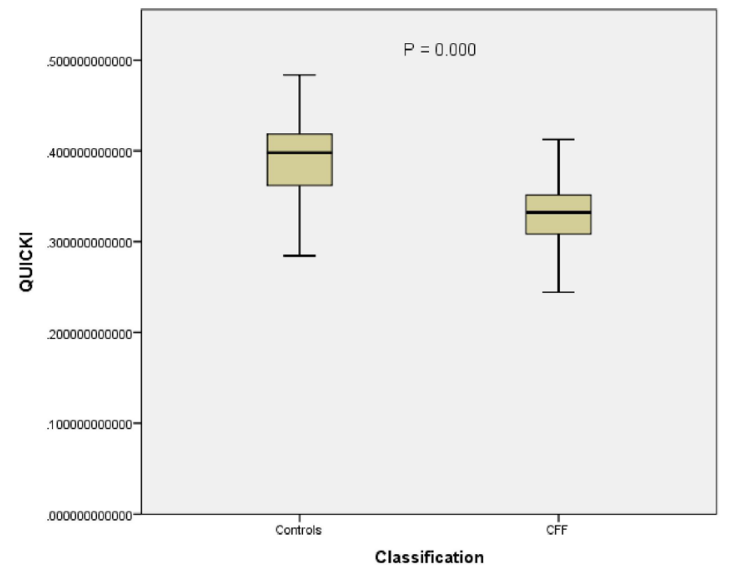

Figure 2 Quantitative Insulin Sensitivity Check Index (QUICKI) in CFF and controls

The observed significant elevation in body weight, BMI, WC, HC, WHR and NC in participants with CFF suggests that individuals with CFF are at more risk of developing diseases associated with central adiposity and obesity. These diseases include type 2 diabetes mellitus and cardiovascular diseases among others. This allusion is further supported by the observed significant positive correlation between CFF thickness and TG as well as WC which are vital components of the metabolic syndrome. Although there is the dearth of information on CFF and cardio-metabolic diseases, the reports of Preis et al..$^{7}$ and Stabe et al. ${ }^{8}$ showed that neck circumference (NC), is an independent 


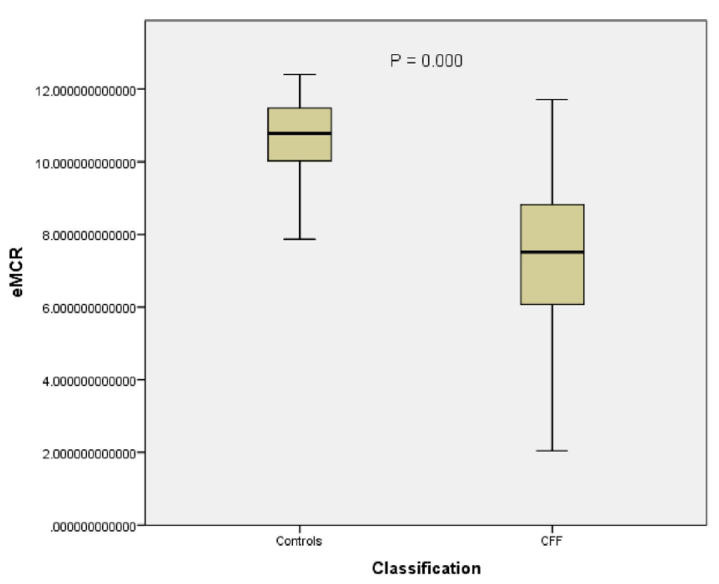

Figure 3 Estimated metabolic clearance rate of glucose in CFF and controls

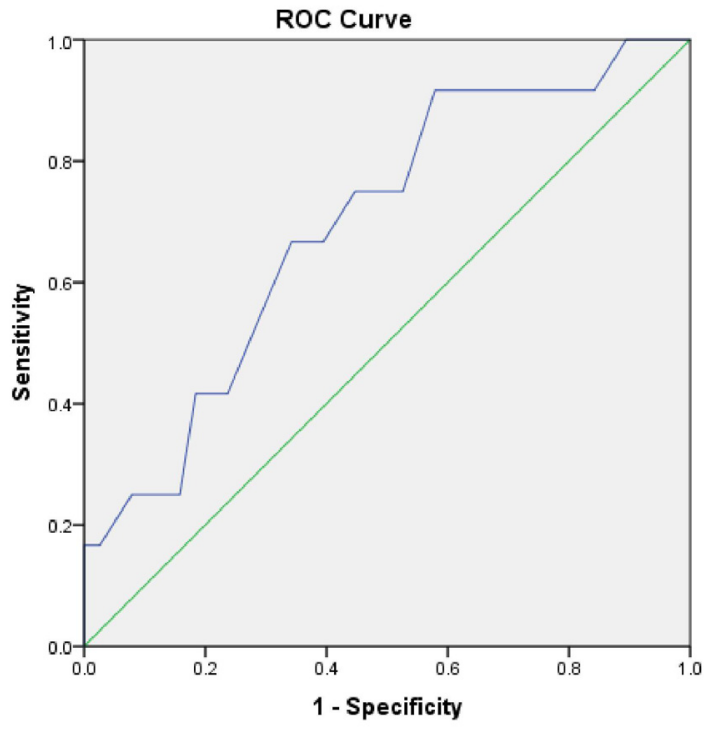

Figure 4 ROC curve of CFF thickness in men with Normal Insulin Sensitivity status and men with Low Insulin Sensitivity status

predictor of cardiovascular disease risk beyond BMI and WC. They reported that large NC is associated with various cardio-metabolic disruptions even after adjusting for VAT and body mass index (BMI). These reports clearly show that accumulation of fat in the upper segment of the body such as the neck may confer risk above and beyond visceral abdominal fat accumulation.

Insulin resistance is not simply a problem of hyper-insulinaemia with its attendant hyperglycaemia, but a complex syndrome that increases significantly the risk for CVDs. ${ }^{16,19}$ Over the years, simple surrogate indices of insulin resistance/ sensitivity including HOMA-IR, QUICKI and eISI among others have been used to appropriately explore the epidemiology, pathophysiological mechanisms, outcomes of therapeutic interventions, and clinical courses of patients with insulin resistance. ${ }^{13}$ The observed elevation in HOMA-IR and FIRI values and the concurrent reduction in QUICKI and eISI values indicate that males with CFF have impaired response to insulin action which could culminate in hyperglycaemia. As a result of IR, the pancreas attempts to compensate by secreting increasing amounts of insulin, resulting in hyperinsulinemia. ${ }^{20}$ This explains the observed elevated levels of FI and 2 hour PPI in men with. CFF. Despite the observed IR in men with CFF, FPG and 2 hour PPG did not differ significantly from men without CFF. This is not surprising as glucose tolerance is maintained via a series of IR activated physiological changes including compensatory hyperinsulinaemia. ${ }^{21}$

Metabolic clearance rate (MCR) of glucose is a predictor of insulin sensitivity status and its reduction indicates low glucose disposal rate, a forerunner of glucose intolerance. ${ }^{22-24}$ Akinlade et al. ${ }^{25}$ showed that reduction in eMCR of glucose increases the risk of glucose intolerance. This supports the observed low level of eMCR in men with CFF which corroborates the observed IR and reduced IS in men with CFF. These observations indicate that men with CFF have increased predisposition to metabolic dysregulation with its associated diseases.

Previous studies ${ }^{7,8}$ showed that positive correlation exists between neck circumference and insulin resistance (IR) as well as biochemical components of the metabolic syndrome (MS). In this study, similar observation was made as CFF thickness had positive correlation with indices of adiposity (BMI and WC) and insulin resistance (HOMA-IR and FIRI) but a negative correlation with QUICKI. These observations buttress the fact that men with CFF have increased risk of developing obesity-cum-IR associated diseases.

We sought to know if CFF thickness could predict insulin sensitivity status in men with CFF and it was observed that CFF thickness has $69.5 \%$ comparability to the QUICKI cut-off values. A cut-off value of $12.5 \mathrm{~cm}$ of CFF thickness was found to have a sensitivity of $75.0 \%$ and a specificity of $55.3 \%$ in indicating LIS. This suggests that CFF thickness of $12.5 \mathrm{~cm}$ and above could indicate LIS status in men with CFF. Our observation indicates that CFF thickness is not just an indication of fat deposition but more importantly, a marker of IR.

Small sample size is a limitation in this study hence, a population study is suggested to confirm the findings from this study.

It could be concluded from this study that adult males with CFF have insulin resistance and low metabolic clearance rate of glucose and are thus, prone to developing cardiovascular and metabolic 
diseases. Also, CFF thickness appears to be a good predictor of insulin resistance.

\section{FUNDING}

Self-funded.

\section{CONFLICT OF INTEREST}

Authors have no competing interest to declare.

\section{AUTHORS CONTRIBUTION}

KSA designed the study, all authors recruited the study participants, AOA did the laboratory analysis, KSA and SKR did the metabolic calculation, statistical analysis and wrote the draft of the manuscript, all the authors edited and approved of the final draft of the manuscript, KSA supervised the entire research.

\section{REFERENCES}

1. Małodobra-Mazur M, Alama A, Bednarska-Chabowska D, Pawelka D, Myszczyszyn A, Dobosz T. Obesity-induced Insulin Resistance via Changes in the DNA Methylation Profile of Insulin Pathway Genes. Adv Clin Exp Med 2019; 28(12):1599-1607. doi: 10.17219/acem/110321.

2. Shulman GI. Ectopic fat in insulin resistance, dyslipidemia, and cardiometabolic disease. N Engl J Med 2014; 371(12):1131-1141. doi: 10.1056/NEJMra1011035.

3. Liu L, Feng J, Zhang G, Yuan X, Li F, Yange T et al. Visceral adipose tissue is more strongly associated with insulin resistance than subcutaneous adipose tissue in Chinese subjects with pre-diabetes. Curr Med Res Opin 2018; 34(1):123-129. doi: 10.1080/03007995.2017.1364226.

4. Lalia AZ, Dasari S, Johnson ML, Robinson MM, Konopka AR, Distelmaier K et al. Predictors of WholeBody Insulin Sensitivity Across Ages and Adiposity in Adult Humans. J Clin Endocrinol Metab 2016; 101(2):626634. doi: 10.1210/jc.2015-2892.

5. Gonzalez N, Moreno-Villegas Z, Gonzalez-Bris A, Egido J, Lorenzo Ó. Regulation of visceral and epicardial adipose tissue for preventing cardiovascular injuries associated to obesity and diabetes. Cardiovasc Diabetol 2017;16(1):44. doi: 10.1186/s12933-017-0528-4.

6. Nielsen S, Guo Z, Johnson CM, Hensrud DD, Jensen MD. Splanchnic lipolysis in human obesity. J Clin Invest 2004; 113(11):1582-1588. doi: 10.1172/JCI21047.

7. Preis SR, Massaro JM, Hoffmann U, D’Agostino Sr RB, Levy D, Robins SJ et al. Neck circumference as a novel measure of cardiometabolic risk: the Framingham Heart study. J Clin Endocrinol Metab 2010; 95(8):3701-3710. doi: 10.1210/jc.2009-1779.

8. Stabe C, Vasques ACJ, Lima MMO, Tambascia MA, Pareja JC, Yamanaka A et al. Neck circumference as a simple tool for identifying the metabolic syndrome and insulin resistance: results from the Brazilian Metabolic Syndrome Study. Clin Endocrinol (Oxf) 2013; 78(6):874881. doi: 10.1111/j.1365-2265.2012.04487.x.

9. Britton KA, Massaro JM, Murabito JM, Kreger BE, Hoffmann U, Fox CS. Body fat distribution, incident cardiovascular disease, cancer, and all-cause mortality. J Am Coll Cardiol 2013; 62(10):921-925. doi: 10.1016/j. jacc.2013.06.027.

10. Kihara S, Matsuzawa Y. Fat distribution and cardiovascular disease risk. Curr Cardiovasc Risk Rep 2015; 9(3):8. https://doi.org/10.1007/s12170-015-0439-4.
11. Fan $\mathrm{H}$, Li X, Zheng L, Chen X, Lan $\mathrm{Q}, \mathrm{Wu} \mathrm{H}$ et al. Abdominal obesity is strongly associated with Cardiovascular Disease and its Risk Factors in Elderly and very Elderly Community-dwelling Chinese. Sci Rep 2016; 6:21521. doi: 10.1038/srep21521.

12. Friedewald WT, Levy RI, Fredrickson DS. Estimation of the concentration of low-density lipoprotein cholesterol in plasma, without use of the preparative ultracentrifuge. Clin Chem 1972; 18(6):499-502.

13. Muniyappa R, Lee S, Chen H, Quon MJ. Current approaches for assessing insulin sensitivity and resistance in vivo: advantages, limitations, and appropriate usage. Am J Physiol Endocrinol Metab 2008; 294(1):E15-E26. doi: 10.1152/ajpendo.00645.2007

14. Stumvoll M, Van Haeften T, Fritsche A, Gerich J. Oral glucose tolerance test indexes for insulin sensitivity and secretion based on various availabilities of sampling times. Diabetes Care 2001; 24(4):796-797. doi: 10.2337/ diacare.24.4.796.

15. Keskin M, Kurtoglu S, Kendirci M, Atabek ME, Yazici C. Homeostasis model assessment is more reliable than the fasting glucose/insulin ratio and quantitative insulin sensitivity check index for assessing insulin resistance among obese children and adolescents. Pediatrics 2005; 115(4):e500-503. doi: 10.1542/peds.2004-1921.

16. Ginsberg HN. Insulin resistance and cardiovascular disease. J Clin Invest 2000; 106(4):453-458. doi: 10.1172/ JCI10762.

17. Argiles JM, Lopez-Soriano FJ. Insulin and cancer. Int J Oncol 2001; 18(4):683-687. https://doi.org/10.3892/ ijo.18.4.683

18. Zhang Y, Lee ET, Howard BV, Best LG, Umans JG, Yeh J et al. Insulin resistance, incident cardiovascular diseases, and decreased kidney function among nondiabetic American Indians: the Strong Heart Study. Diabetes Care 2013; 36(10):3195-3200. doi: 10.2337/dc12-2368.

19. McFarlane SI, Banerji M, Sowers JR. Insulin Resistance and Cardiovascular Disease. J Clin Endocrinol Metab 2001; 86(2):713-718. doi: 10.1210/jcem.86.2.7202.

20. Ferreira AP, Oliveira C, França NM. Metabolic syndrome and risk factors for cardiovascular disease in obese children: the relationship with insulin resistance (HOMA-IR). J Pediatr (Rio J) 2007; 83(1):21-26. doi: 10.2223/JPED.1562.

21. Wilcox G. Insulin and insulin resistance. Clin Biochem Rev 2005; 26(2):19.

22. Revers RR, Kolterman OG, Olefsky JM. Relationship between serum glucose level and the metabolic clearance rate of glucose in non-insulin-dependent diabetes mellitus. Diabetes 1983; 32(7):627-632. doi: 10.2337/diab.32.7.627.

23. Stumvoll M, Mitrakou A, Pimenta W, Jenssen T, YkiJärvinen $\mathrm{H}$, Van Haeftene $\mathrm{T}$ et al. Use of the oral glucose tolerance test to assess insulin release and insulin sensitivity. Diabetes Care 2000; 23(3):295-301. doi: 10.2337/ diacare.23.3.295.

24. Lee CC, Haffner SM, Wagenknecht LE, Lorenzo C, Norris JM, Bergman RN et al. Insulin clearance and the incidence of type 2 diabetes in Hispanics and African Americans: the IRAS Family Study. Diabetes Care 2013; 36(4):901-907. doi: 10.2337/dc12-1316.

25. Akinlade KS, Rahamon SK, Lasebikan VO. Beta-cell Function and Metabolic Clearance Rate of Glucose in Patients with Major Mental Health Disorders on Antipsychotic Drug Treatment. J Natl Med Assoc 2018; 110(5):504-511. doi: 10.1016/j.jnma.2018.01.003.

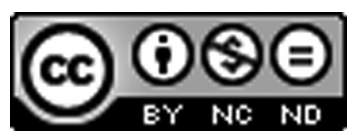

This work is licensed under a Creative Commons Attribution-Non Commercial-No Derivatives 4.0 International License. To view a copy of this license, visit http://creativecommons.org/licenses/by-nc-nd/4.0/ 